\title{
Tissue Attenuation Estimation from Backscattered Ultrasound Using Spatial Compounding Technique - Preliminary Results
}

\author{
Ziemowit KLIMONDA, Jerzy LITNIEWSKI, \\ Andrzej NOWICKI \\ Institute of Fundamental Technological Research \\ Polish Academy of Sciences \\ Pawińskiego 5B, 02-106 Warszawa, Poland \\ e-mail: zklim@ippt.gov.pl \\ (received October 8, 2010; accepted November 15, 2010)
}

\begin{abstract}
The pathological states of biological tissue are often resulted in attenuation changes. Thus, information about attenuating properties of tissue is valuable for the physician and could be useful in ultrasonic diagnosis. We are currently developing a technique for parametric imaging of attenuation and we intend to apply it for in vivo characterization of tissue. The attenuation estimation method based on the echoes mean frequency changes due to tissue attenuation dispersion, is presented. The Doppler IQ technique was adopted to estimate the mean frequency directly from the raw RF data. The Singular Spectrum Analysis technique was used for the extraction of mean frequency trends. These trends were converted into attenuation distribution and finally the parametric images were computed. In order to reduce variation of attenuation estimates the spatial compounding method was applied. Operation and accuracy of attenuation extracting procedure was verified by calculating the attenuation coefficient distribution using the data from the tissue phantom (DFS, Denmark) with uniform echogenicity while attenuation coefficient underwent variation.
\end{abstract}

Keywords: ultrasound attenuation estimation, spatial compounding, parametric imaging.

\section{Introduction}

The ultrasonic imaging is non-invasive, popular and relatively inexpensive method of visualization of the body interior. The USG image consists of many lines; each of them corresponds to the echo envelope. Such imaging mode is called B-mode. The B-mode image reflects the distribution of the tissue reflec- 
tivity, that depends on acoustical impedance variations. However, the raw radiofrequency $(\mathrm{RF})$ echoes contain information about the tissue properties that cannot be assessed with the signal envelope. The attenuation of ultrasound is one of such properties with potentially substantial importance in medical diagnostics. It has been demonstrated that the pathological tissue differs in attenuating properties from the healthy one. OOSTERVELD et al. (1991) have shown that the slope of attenuation coefficient, combined with statistical parameters of image texture can be used to diagnose the diffuse liver disease. SAIJO and SASAKI (1996) employed scanning acoustic microscope to measure five types of gastric cancer and indicated different attenuation coefficients and sound speeds comparing to normal tissue. Bigelow et al. (2008) investigated possibility of prediction of the premature delivery based on the noninvasive ultrasonic attenuation determination. In many other publications it has been reported that pathological processes can lead to changes in the mean attenuation coefficient that range from several percent for cirrhotic human liver, through dozens percent for fatty human liver (Lu et al., 1999), or degenerated bovine articular cartilage (NiEMinen et al., 2004) to over a hundred percent in case of porcine liver HIFU treatment in vivo (ZDERIC et al., 2004) or two hundred percent for porcine kidney thermal coagulation (Worthington, Sherar, 2001). These reports motivated us to consider the parametric imaging of the attenuation as a useful tool in medical diagnostic. There are two approaches to the estimation of the ultrasonic attenuation. The spectral difference technique, that is based on a comparison of the power spectrum of backscattered signals before and after the wave propagation through the medium, and the spectral shift method that uses the downshift of the pulse mean frequency caused by the frequency-depended attenuation. In our approach the mean frequency (MF) is directly evaluated from the backscatter data by means of the correlation estimator. Mean frequency is highly variable due to the random character of the backscattering RF signals (KLImONDA, Nowicki, 2007; OpHIR et al., 1985). In this work the Singular Spectrum Analysis (SSA) algorithm and the spatial compounding technique were used to extract the mean frequency trend and to decrease the random variance of the estimated attenuation profile. The data were collected from the tissue mimicking phantom using the commercial ultrasonic scanners, equipped with the special research modules capable to acquire RF data before envelope detection.

\section{Methods}

We assumed, that the attenuation in tissue can be described by the following model. The amplitude of the wave propagating in tissue decreases exponentially due to attenuation what can be expressed as

$$
A=A_{0} \exp (-\alpha x)
$$


where $A_{0}$ - initial intensity, $\alpha$-attenuation coefficient and $x$ - wave path length. The attenuation coefficient $\alpha$ depends on frequency $f$ and in the soft tissue it has the following form:

$$
\alpha(f)=\alpha_{1}\left(\frac{f}{f_{1}}\right)^{n},
$$

where $\alpha_{1}$ is the attenuation coefficient at the frequency $f_{1}$ (in the literature generally $f_{1}=1 \mathrm{MHz}$ ) and $n$ for the soft tissue is close to 1 (NowICKI, 2000). Thus, the linear relation between attenuation coefficient in tissue and the wave frequency is often assumed. When a short ultrasonic pulse propagates within the homogenous medium the dispersion of the attenuation coefficient results in the shift of the pulse mean frequency (MF). To find estimates of the attenuation from ultrasonic echo signals we assume that the attenuation of tissue increases linearly with frequency and that the backscattered signals have the Gaussian shaped spectra (Gaussian pulses). Then, the MF shift $\left(f-f_{0}\right)$ is given by (LAUGIER, 1985; LitNiEWSKI, 2006)

$$
f-f_{0}=\frac{\alpha \cdot \Delta x \cdot \sigma_{0}^{2}}{2},
$$

where $f_{0}$ and $f$ are MF before and after propagation respectively, $\sigma_{0}^{2}$ is the Gaussian variance of the pulse spectrum, $\Delta x$ denotes penetrated distance and $\alpha$ is the attenuation coefficient. Gaussian pulse spectrum preserves the shape during propagation in linearly attenuating medium, i.e. the $\sigma_{0}^{2}$ is constant, and the $\alpha$ can be calculated from Eq. (3) as

$$
\alpha=-\frac{2}{\sigma_{0}^{2}} \frac{\partial f}{\partial x} .
$$

The attenuation coefficient $\alpha$ is positive, thus MF along the propagation path, i.e. the MF line always decreases monotonically with the penetration depth. To determine the MF line we have applied the MF correlation estimator (I/Q algorithm). The estimator is defined by

$$
\mathrm{MF}=\frac{1}{2 \pi T_{s}} a \tan \left(\frac{\sum_{i=1}^{N} Q(i) I(i+1)-Q(i+1) I(i)}{\sum_{i=1}^{N} I(i) I(i+1)+Q(i+1) Q(i)}\right),
$$

where $T_{s}$ is the sampling period and $N$ is the estimator window length. The $Q$ and $I$ are quadrature and in-phase signal components. The $N$ is parameter of significant importance, because it is directly related to the resolution of the method (KLIMONDA et al., 2009). The $Q$ and $I$ are obtained by quadrature sampling technique. The quadrature sampling is often used in modern scanners and the correlation estimator is widely used in Doppler techniques (NowiCKI, 1995). The presented technique of attenuation estimation consists of four steps. First, 
the raw RF data are filtered with the filter that has bandwidth corresponding to the bandwidth of the transducer. Next, the estimator window moves along the filtered RF line and the MF values are determined. The MF line is created point by point. In ideal case, the attenuation (A) line could be enumerated directly from MF line using the Eq. (4). Unfortunately, the real MF lines are highly variable and the direct use of Eq. (4) results in the highly noised A lines, not acceptable for the attenuation imaging. Thus, in the third step the reduction of the MF line random variability is required. This is realized by the moving average filtration and the Singular Spectrum Analysis (SSA) technique. The moving average filter operating over adjacent MF lines in lateral direction is applied. Then the SSA trend extraction algorithm is employed. In the last step the Eq. (4) is applied and the A lines are enumerated from the smoothed MF lines. Finally, the spatial compounding technique is used.

The SSA is relatively new technique of analysis of the time series. The aim of this technique is the decomposition of the input data series into the sum of components which can be interpret as the trend, oscillatory components and the noise (non-oscillatory components). The major applications of the SSA technique is the smoothing of the time series, finding the trend, forecasting and detection of the structural changes (Alonso et al., 2005; Golyandina et al., 2007; Hassani, 2007; Moore, Grinsted, 2006; Schoellhamer, 2001; VAradi et al., 2000; VAUTARD, 1992). The SSA is the model-free technique - there is no need to know a general function describing how the MF changes with the depth. Another useful feature of the SSA is its robustness to the outliers (AlEXANDROV 2009). The SSA is easy to use - it needs only one parameter - the window length. Details of this technique can be found in the literature (GOLYANDINA et al., 2001). The application of the SSA and the averaging of the scan lines limits the variations of the attenuation estimate but it is still affected by errors. In this approach, the spatial compounding technique was used to increase accuracy of the attenuation estimate and to reduce its variations. The spatial compounding is often applied to reduce speckles in standard B-mode images (BEHAR et al., 2003). The method bases on averaging images obtained from different but closely located scan planes. The variation of the scanning plane position can be realized by shifting the probe perpendicularly to the scan plane (Fig. 1a) or by the slight modification of the imaging plane angle (Fig. 1b). In this paper the compounding technique that is often used in standard B-mode imaging was applied to the attenuation distribution imaging.

\section{Experimental setup}

The experimental setup consisted of the ultrasonic scanner (ZONARE z.one ultra SmartCart System or SIEMENS ANTARES) and the tissue mimicking phantom produced by Dansk Fantom Design. The Zonare and the Siemens scan- 
ners were equipped with linear VF13-5 and the convex CH6-2 probe, respectively. The pulse frequency were $5 \mathrm{MHz}$ (linear) and $4.44 \mathrm{MHz}$ (convex). The scanners were equipped with the special research modules (IQscan and Axius Direct Ultrasound Research Interface respectively) which enable an access to the unprocessed $\mathrm{RF}$ data. The RF data were recorded during the scanning, transmitted to PC computer, and processed offline with the Matlab ${ }^{\circledR}$ software. The attenuation of the phantom background was equal to $0.7 \mathrm{~dB} /(\mathrm{MHz} \cdot \mathrm{cm})$. It contained the cylindrical volume of the $1.5 \mathrm{~cm}$ diameter with the attenuation coefficient equal to $1.1 \mathrm{~dB} /(\mathrm{MHz} \cdot \mathrm{cm})$. The cylinder was localized at the depth of $5 \mathrm{~cm}$ and its echogenicity was similar to the echogenicity of the background.

Two compounding schemes was applied. First the probe was by moved along the cylinder axis and the successive scans with $1 \mathrm{~mm}$ intervals between scan planes were collected (Fig. 1a). The number of scans equaled to twelve and acquisition was realized with linear probe. In the second compounding scheme the variation of the angle between the cylinder axis and the probe axis (Fig. 1b) was applied. The number of scans equaled to six. The acquisition was realized with the convex probe. The probe was operated by hand, so the exact angle changes are unknown, but the B-mode image was controlled during the measurements. There were no noticeable changes in the shape of the cylinder's projection on the scan plane, so the assumption of small angle changes is justified.

a)

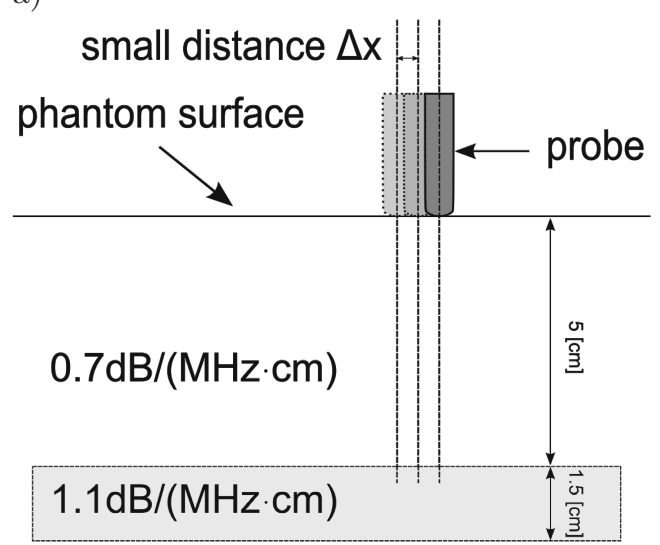

b)

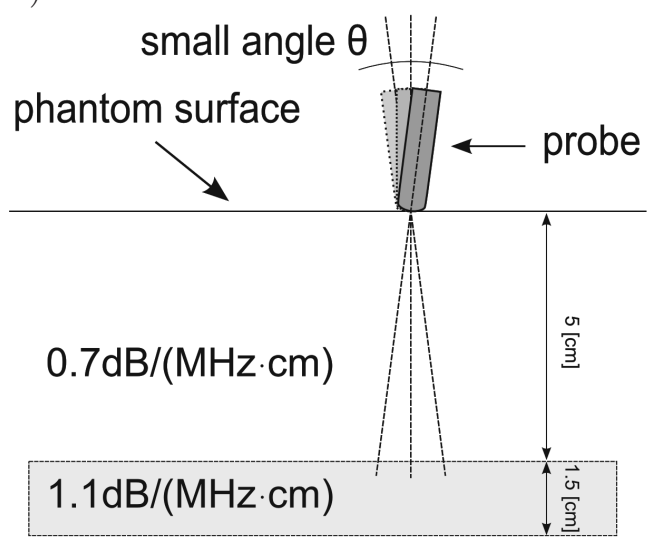

Fig. 1. Two schemes of compounding scanning linear (a) and convex (b) probe.

Each scan was processed using algorithm, depicted in the METHODS section, to obtain attenuation estimate. The correlation estimator window and the SSA window length corresponded to $10 \mathrm{~mm}$ depth for linear probe and $20 \mathrm{~mm}$ for convex probe. The final attenuation image was created by averaging component estimates. Additionally, twelve RF scans of the phantom background, with attenuation equal to $0.7 \mathrm{~dB} /(\mathrm{MHz} \cdot \mathrm{cm})$ were recorded. These data were used to evaluate the coefficient $2 / \sigma_{0}^{2}$ of the Eq. (4). 


\section{Results}

The Fig. 2 presents the comparison between the standard B-mode image and the attenuation image produced using spatial compounding. The volume with higher attenuation is localized in the centre of the scan. On the B-scan (Fig. 2a) the presence of the high attenuation area is manifested by the "shadow" below this area but it is hardly visible. However, in the attenuation image (Fig. 2b) this volume is clearly visible. The estimate matches the attenuation coefficients of the tissue phantom very well. The estimated values varies from 0.6 to $0.8 \mathrm{~dB} /(\mathrm{MHz} \cdot \mathrm{cm})$ and from 1.0 to $1.1 \mathrm{~dB} /(\mathrm{MHz} \cdot \mathrm{cm})$ in the background area and the centre of the high attenuation cylinder volume, respectively.

a)

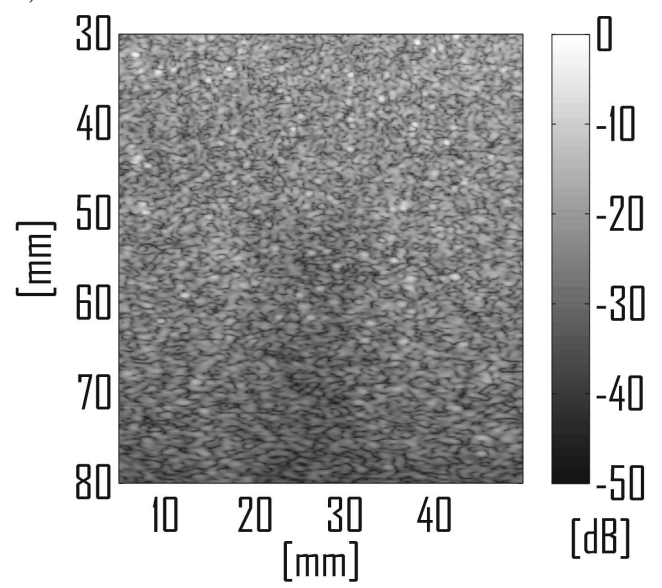

b)

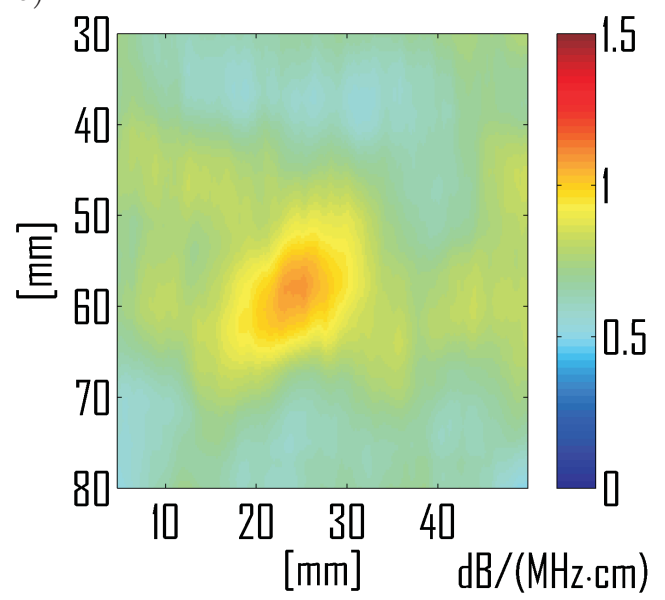

Fig. 2. The standard B-mode image (a) and the image of the attenuation distribution using spatial compounding technique (b), obtained by linear probe.

The components of the compounded attenuation image are of worse quality than the final estimate. The object is visible in four component images however strong noise is also present. The object is almost undetectable in the rest of the component images. The Fig. 3 presents two of them. The left image (Fig. 3a) is the best of four scans which show the object clearly. The right (Fig. 3b) represents the group of images where the object is hardly visible. The values of estimated attenuation in Fig. 3a varies from 0.1 to $1.0 \mathrm{~dB} /(\mathrm{MHz} \cdot \mathrm{cm})$ and from 1.0 to $1.5 \mathrm{~dB} /(\mathrm{MHz} \cdot \mathrm{cm})$ in the background area and in the highly attenuating cylinder volume, respectively.

The results obtained for convex probe are presented in Fig. 4. The attenuating object is clearly visible (Fig. $4 \mathrm{~b}$ ). The attenuation value corresponding to the axis of the cylinder is equal to $1.0 \mathrm{~dB} /(\mathrm{MHz} \cdot \mathrm{cm})$. The highly attenuating areas on the upper edge of the image are fake and probably results from spectrum changes produced by the crossing the probe-phantom interface. The area in the lower 
a)

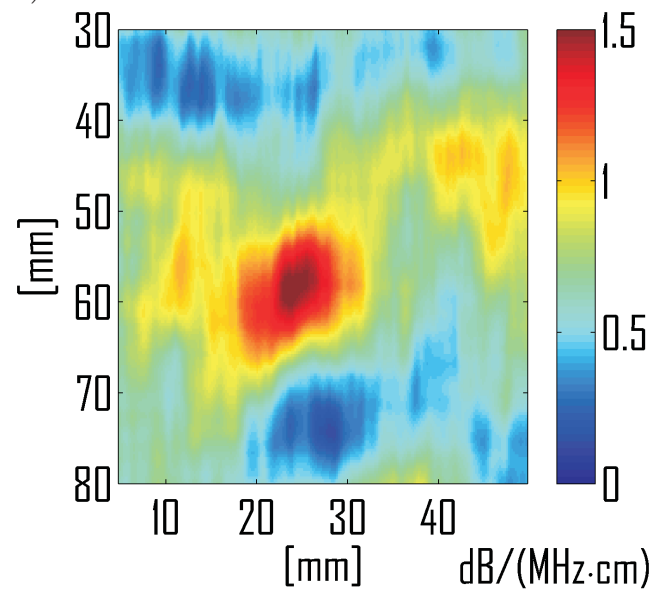

b)

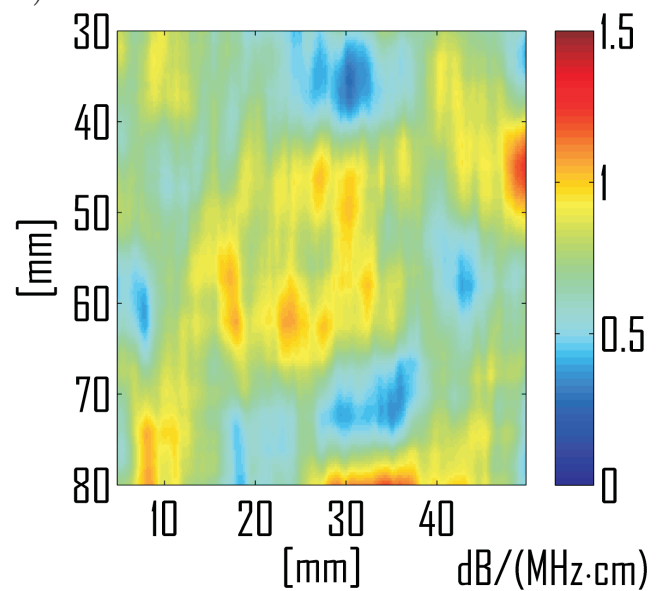

Fig. 3. Two components of the compounded attenuation image, the one with well visible object (a), and the other with hardly visible object obtained with linear probe.

a)

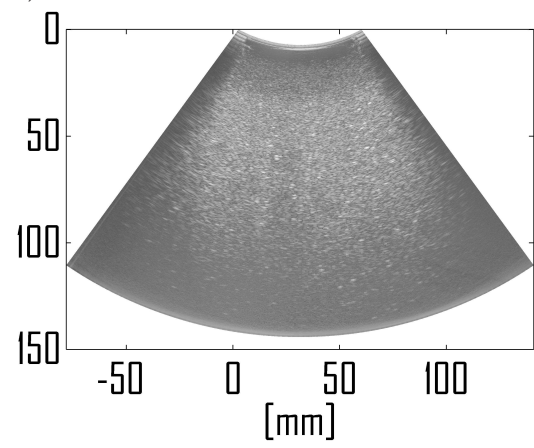

b)

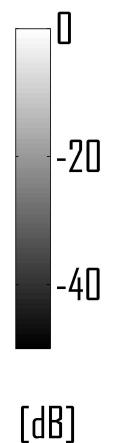

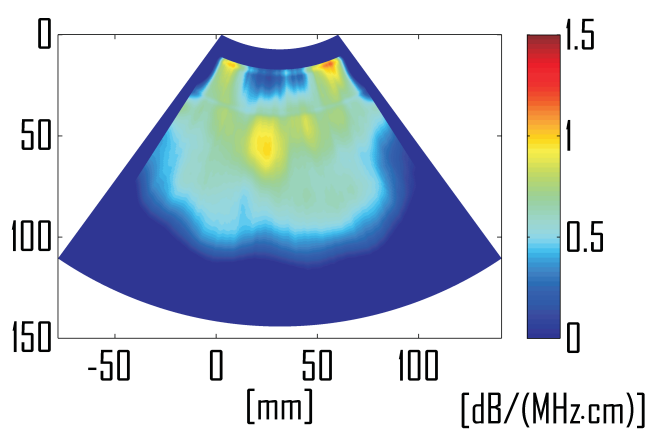

Fig. 4. The standard B-mode image (a) and the image of the attenuation distribution using spatial compounding technique (b), obtained by convex probe.

part of the image was estimated as equal to $0 \mathrm{~dB} /(\mathrm{MHz} \cdot \mathrm{cm})$ because of low signal amplitude in this region which is visible in Fig. 4a.

\section{Conclusions}

The method of tissue attenuation estimation is presented. It is based on tracking the mean frequency changes of the backscattered signals. The results indicate, that the spatial compounding technique increases the precision of the method. The compounded estimate matches adequately the attenuation values of the phantom, while the most of the single scans do not. Even if the single scan provides sufficient visibility of the high attenuating area, the estimate is inaccurate. The high attenuating area is hard to detect in the ordinary B-mode 
presentation, while in the attenuation image it is clearly visible. The attenuation images seems to be promising tool in medical diagnostics, however the precision of a single scan is often unsatisfactory. The presented results suggest that the spatial compounding technique is an effective procedure of increasing the precision of the attenuation estimates and reducing of the noise level.

\section{Acknowledgments}

This work was partly supported by the Polish Ministry of Science and Education, project NN518388234.

Preliminary results of this study were presented at the Open Seminar on Acoustics, Gliwice, September 20-24, 2010.

\section{References}

1. Alexandrov T. (2009), A Method of Trend Extraction using Singular Spectrum Analysis, REVSTAT Statistical Journal, 7, 1, 1-22.

2. Alonso F.J., Del Castillo J.M., Pintado P. (2005), Application of singular spectrum analysis to the smoothing of raw kinematic signals, Journal of Biomechanics, 38, 1085-1092.

3. Behar V., Adam D., Friedman Z. (2003), A new method of spatial compounding imaging, Ultrasonics, 41, 377-384.

4. Bigelow T.A., Mcfarlin B.L., O’Brien W.D., Oelze M.L. (2008), In vivo ultrasonic attenuation slope estimates for detectiong cervical ripening in rats: Preliminary results, Journal of Acoustical Society of America, 123, 3, 1794-1800.

5. Golyandina N., Nekrutkin V., Ahigluavsky A. (2001), Analysis of time Series Structure: SSA and related techniques, Chapman \& Hall/CRC.

6. Golyandina N.E, Usevich K.D., Florinsky I.V. (2007), Filtering of Digital Terrain Models by Two-Dimensional Singular Spectrum Analysis, International Journal of Ecology \& Development, 8, f07, 81-94.

7. Hassani H. (2007), Singular Spectrum Analysis: Methodology and Comparison, Journal of Data Science, 5, 239-257.

8. Klimonda Z., Litniewski J., Nowicki A. (2009), Spatial resolution of attenuation imaging, Archives of Acoustics, 34, 4, 461-470.

9. Klimonda Z., Nowicki A. (2007), Imaging of the mean frequency of the ultrasonic echoes, Archives of Acoustics, 32, 4 (Supplement), 77-80.

10. Laugier P., Berger G., Fink M., Perrin J. (1985), Specular reflector noise: effect and correction for in vivo attenuation estimation, Ultras. Imag. 7, 277-292.

11. Litniewski J. (2006), Assessment of trabecular bone structure deterioration by ultrasound, [in Polish: Wykorzystanie fal ultradżwiękowych do oceny zmian struktury kości gąbczastej], Prace IPPT.

12. Lu Z.F., Zagzebski J., Lee F.T. (1999), Ultrasound Backscatter and Attenuation in Human Liver With Diffuse Disease, Ultrasound in Med. \& Biol., 25, 7, 1047-1054. 
13. Moore J.C., Grinsted A. (2006), Signular spectrum analysis and envelope detection: methods of enhancing the utility of ground-penetrating radar data, Journal of Glaciology, $\mathbf{5 2}, 176$.

14. Nieminen H.J., SaArakkala S., LaAsanen M.S., Hirvonen J., Jurvelin J.S., TÖYrÄs J. (2004), Ultrasound Attenuation in Normal and Spontaneously Degenerated Articular Cartilage, Ultrasound in Med. \& Biol., 30, 4, 493-500.

15. Nowicki A. (1995), Fundamentals of Doppler Ultrasonography [in Polish: Podstawy ulrasonografii dopplerowskiej], PWN, Warszawa.

16. Nowicki A. (2000), Ultrasonic Diagnostics [in Polish: Diagnostyka ultradźwiękowa], MAKmed, Gdańsk.

17. Oosterveld B.J, Thijssen J.M., Hartman P.C., Romijn RL., Rosenbusch G.J. (1991), Ultrasound attenuation and texture analysis of diffuse liver disease: methods and preliminary results, Phys. Med. Biol., 36, 8, 1039-1064.

18. Ophir J., Ghouse M.A., Ferrari L.A. (1985), Attenuation estimation with the zero crossing technique: phantom studies, Ultras. Imag., 7, 122-132.

19. Saijo Y., Sasaki H. (1996), High Frequency Acoustic Properties of Tumor Tissue. [in:] Ultrasonic Tissue Characterization, Dunn F., Tanaka M., Ohtsuki S., Saijo Y. [Eds.], 217230 Springer-Verlag Tokio, Hong-Kong.

20. Schoellhamer D.H. (2001), Singular spectrum analysis for time series with missing data, Geophysical Research Letters, 28, 16, 3187-3190.

21. Varadi F., Ulrich R.K., Bertello L., Henney C.J. (2000), Random lag singular cross-spectrum analysis, The Astrophysical Journal, 528, 1.

22. Vautard R., Yiou P., Ghil M. (1992), Singular-spectrum analysis: a toolkit for short, noisy chaotic signals, Physica D, 58, 95-126.

23. Worthington A.E., Sherar M.D. (2001), Changes in Ultrasound Properties of Porcine Kidney Tissue During Heating, Ultrasound in Med. \& Biol., 27, 5, 673-682.

24. Zderic V., Keshavarzi A., Andrew A.M., Vaezy S., Martin R.W. (2004), Attenuation of Porcine Tissues In Vivo After High Intensity Ultrasound Treatment, Ultrasound in Med. \& Biol., 30, 1, 61-66. 